Int. J. Dev. Biol. 57: 821-828 (2013)

doi: $10.1387 /$ ijdb.130213ym

\title{
Dual embryonic origin of the hyobranchial apparatus in the Mexican axolotl (Ambystoma mexicanum)
}

\author{
ASYA DAVIDIAN ${ }^{1}$ and YEGOR MALASHICHEV*,1,2 \\ ${ }^{1}$ Department of Embryology and ${ }^{2}$ Department of Vertebrate Zoology, Faculty of Biology and Soil Sciences, \\ Saint-Petersburg State University, St. Petersburg, Russia.
}

\begin{abstract}
Traditionally, the cartilaginous viscerocranium of vertebrates is considered as neural crest (NC)-derived. Morphological work carried out on amphibian embryos in the first half of the XX century suggested potentially mesodermal origin for some hyobranchial elements. Since then, the embryonic sources of the hyobranchial apparatus in amphibians has not been investigated due to lack of an appropriate long-term labelling system. We performed homotopic transplantations of neural folds along with the majority of cells of the presumptive NC, and/or fragments of the head lateral plate mesoderm (LPM) from transgenic GFP+ into white embryos. In these experiments, the NC-derived GFP+ cells contributed to all hyobranchial elements, except for basibranchial 2, whereas the grafting of GFP+ head mesoderm led to a reverse labelling result. The grafting of only the most ventral part of the head LPM resulted in marking of the basibranchial 2 and the heart myocardium, implying their origin from a common mesodermal region. This is the first evidence of contribution of LPM of the head to cranial elements in any vertebrate. If compared to fish, birds, and mammals, in which all branchial skeletal elements are NC-derived, the axolotl (probably this is true for all amphibians) demonstrates an evolutionary deviation, in which the head LPM replaces NC cells in a hyobranchial element. This implies that cells of different embryonic origin may have the same developmental program, leading to the formation of identical (homologous) elements of the skeleton.
\end{abstract}

KEY WORDS: neural crest, head lateral plate mesoderm, branchial arch, viscerocranium, basibranchial 2

\section{Introduction}

Viscerocranium of jawed vertebrates is organized into arches and includes the jaws and hyobranchial apparatus. The hyobranchial apparatus of lower vertebrates consists of a hyoid arch (visceral arch 2) and a set of post-hyoid pharyngeal (branchial) arches, which number varies. The fullest set of elements of each pharyngeal arch consists of four paired (dorsal to ventral: pharyngobranchials, epibranchials, ceratobranchials, and hypobranchials), and one unpaired one, the basibranchial. In modern salamanders, the dorsal elements of each pharyngeal arch disappear, and the posterior arches undergo even a greater reduction. It is accepted (Reilly and Lauder, 1988) that the typical salamander hyobranchial apparatus has one hyoid arch and four pharyngeal arches consisting of only ventral paired elements cerato- and hypobranchials (cerato- and hypohyals), and two midline elements-unpaired basibranchials 1 and 2 (Fig. 1).

It is widely accepted nowadays, that the vertebrate visceral skeleton and a part of the neurocranium originates from the mes- enchyme of the NC, while some cranial elements are considered or suspected as being of mesodermal or mixed origin (Gross and Hanken, 2008, Seufert and Hall, 1990). This knowledge was initially based on morphological difference between the two types of mesenchymal cells (of NC or mesodermal origin), including size, differential staining, pigmentation, and number and size of yolk granules; e.g., the former are small, pigmented, slightly loaded with the yolk granules and dark staining, while the latter are large, non-pigmented, heavily yolk-loaded and lightly staining (Hörstadius, 1950, Landacre, 1921). However, morphological identification of cells from the two sources is mostly possible in the cell undifferentiated (mesenchymal) state, while later NC and mesoderm cells loose their differential qualities (Stone, 1926). This makes it difficult to determine not only the main embryonic source of cells, but also the fact of a possible mixture of cells of two embryonic anlagen

Abbreviations used in this paper: GFP, green fluorescent protein; LPM, lateral plate mesoderm; NC, neural crest.

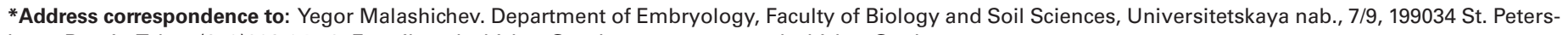
burg, Russia.Tel: +7(812)328-94-53. E-mail: malashichev@embryo.pu.ru or y.malashichev@spbu.ru
} 
within a single cartilaginous element. Interspecies xenotransplants, in which the transplanted cells are mainly differentiated by size, would mostly be now considered not very precise and reliable, as well (Gross and Hanken, 2008).

Extirpation and ectopic transplantation experiments served as additional sources of information. A number of studies in the first half of the $X X$ century reported deficiencies in the viscerocranium after extirpation of fragments of the neural folds or streams of NC cells migrating under the ectoderm to the pharyngeal region in several species of anuran and urodelan amphibians (Bombina, Rana, Ambystoma) or, alternatively, development of ectopic cartilages after heterotopic transplantation of portions of the NC alone or together with parts of the ectoderm and the dorsal neural tube (Hörstadius, 1950, Landacre, 1921, Raven, 1931, Stone, 1926, Stone, 1929). Based on morphological descriptive work and such experimental approaches all the authors concluded that the majority of the viscerocranium cartilages in amphibians are of NC origin, particularly the jaw, hyoid, and branchial arches. However, some viscerocranial cartilages still developed even under conditions of severe extirpations of the NC precursors, so that extirpations led only to reduction in size, not in disappearance of a number of elements (Stone, 1926). In addition, unilateral head lateral plate mesoderm (LPM) transplantation, with both ecto- and endoderm included into the graft, from the head to the place of trunk somites in Ambystoma punctatum embryos at stages before the launch of neural crest migration led to development of ectopic rod-like cartilages, suggesting their probable non-NC (mesodermal?) origin (Stone, 1932). Extirpation (especially in urodelan amphibians, which possess a strong degree of regeneration capacities) is not a reliable approach for fate mapping. On the other hand, the direct
Fig. 1. Results of transplantations of fragments of a neural fold. (A) Schematics demonstrating orthotopical grafting of GFP+ neural folds (including neural crest) from a GFP+ neurula (green, stage 16) into a white (d/d) host. Both entire GFP+ neural folds were grafted into a white host whose neural folds from both sides had been removed before. (B) Schematics demonstrating one-sided orthotopical grafting of a long left GFP + neural fold fragment (including neural crest) into a white (d/d) host. The graft is extirpated from a GFP+ neurula (green, stage 16) and extends from posterior head to anterior trunk. It is implanted into a white host where a similarly sized fragment was extirpated before. (C) Schematics illustrating the anatomical structure of the hyobranchial apparatus, position of the heart (h), and the summary of the transplantations (green - elements marked with GFP after neural fold grafting, blue-basibranchial 2 (bbr2), which has never been marked). Green box around the heart indicates contribution of the neural crest to the septae and truncus arteriosus. The schematic corresponds to (D) ventral aspect of a two months old juvenile chimera carrying a two long GFP+ neural fold fragment. Neural crest cells label upper and lower jaws (ja), nearly complete hyobranchial apparatus, with exception of the bbr2 (no NC labelling) - note the absents of the GFP+ labelling in the region on the midline in front of the heart. (E-G) Right side of a juvenile chimera after unilateral neural fold transplantation-NC derivatives are marked with GFP+ cells and shown at transverse sections. Section plane is shown as on (C) with a dashed line $(E-G),(F, G)$ - enlarged area shown with
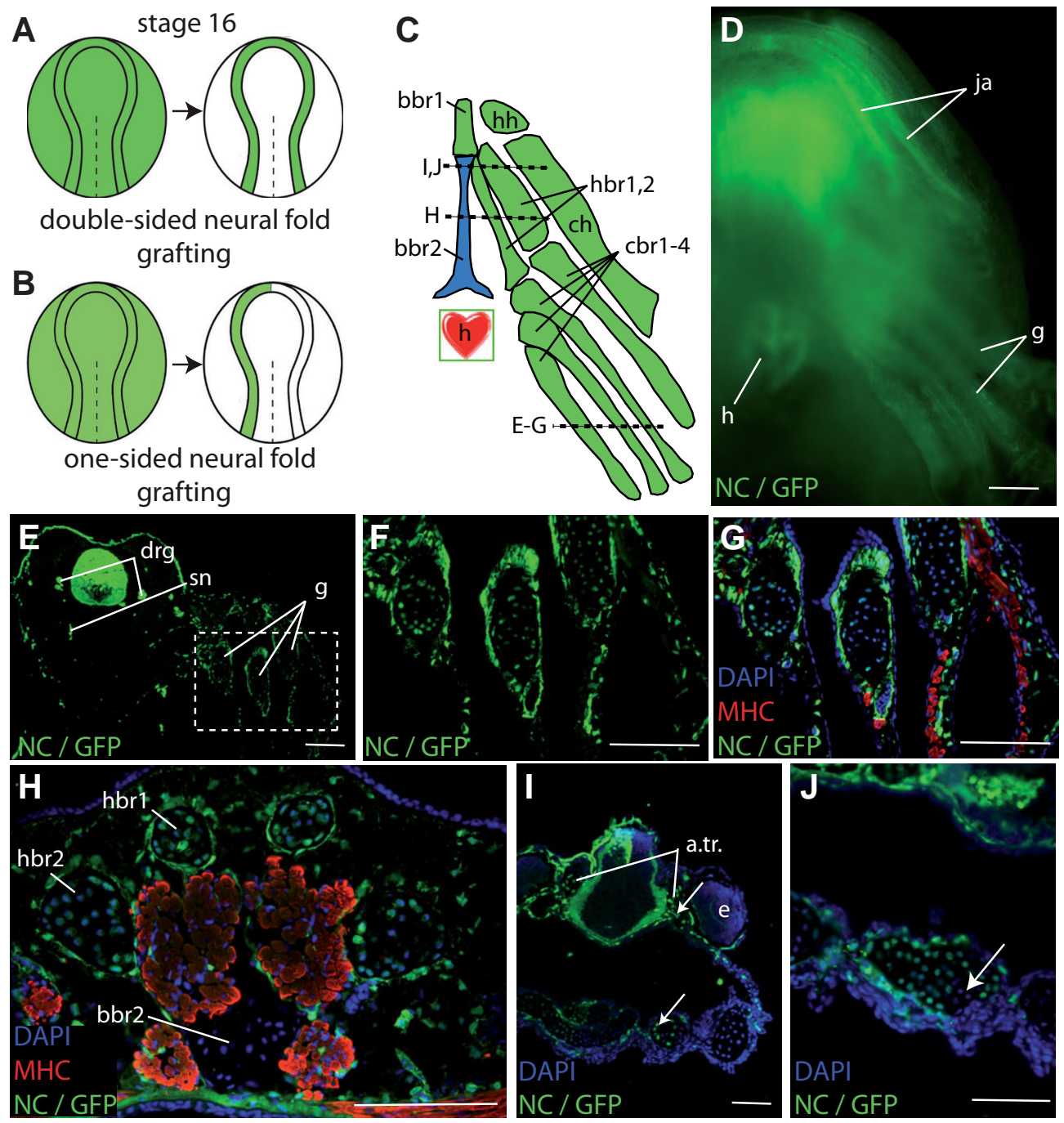

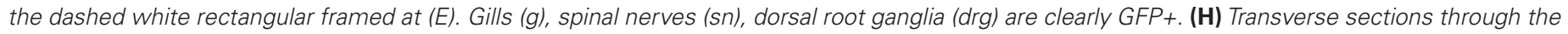

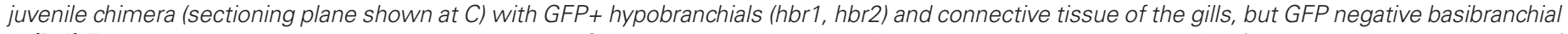

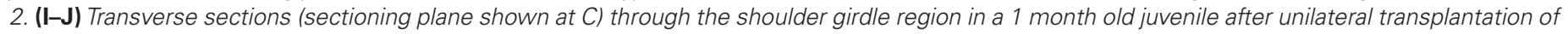

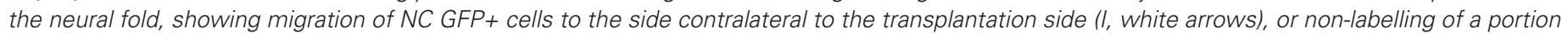

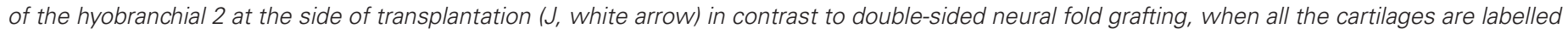

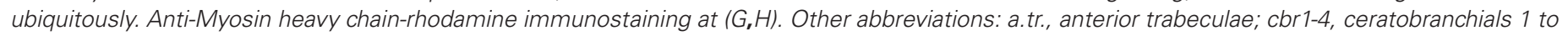
4; ch, ceratohyal; e, eye; h, heart; hbr1,2, hypobranchials 1 and 2; hh, hypohyal. Scale bars: D, 1 mm; E-J: 100 um. 
labelling of mesoderm with homotopic tissue transplantations in axolotls or other amphibians to uncover the actual embryonic origin of each element in the hyobranchial apparatus has never been persuade, because of the lack of an appropriate labelling system.

Recent advances in transgenic techniques led to establishing a number of transgenic lines of amphibians (e.g., Mexican axolotl, A. mexicanum) expressing genes of fluorescent proteins in all cells of the organism, suitable for cell lineage labelling experiments. By using transgenic axolotl system we aimed to explore the origin of the hyobranchial apparatus in axolotl in more detail by transplantation of either neural crest or mesoderm, or both from transgenic GFP+ (Sobkow et al., 2006), or Cherry+ (Kragl et al., 2009) embryos to white host embryos and following up the fate of the transplanted tissues until the formation of the visceral cartilages. Here we show for the first time that the second basibranchial, but not any other visceral cartilages, originates in axolotl from the most ventral part of the head LPM, thus demonstrating dual NC and mesodermal origin for the hyobranchial apparatus. We further discuss evolutionary and developmental aspects of the viscerocranium in amphibians.

\section{Results}

\section{Neural crest contributes to the majority of viscerocranium, but not to basibranchial 2}

To determine NC contribution to the viscerocranium in axolotl, we firstly have used double-sided (Fig. 1A) or one-sided (Fig. 1B) neural fold grafting (most anterior head to posterior trunk region) including NC cells from all possible cranio-caudal levels from a GFP+ donor to a white (d/d) host as shown before (Epperlein et al., 2012). This kind of transplantation resulted in GFP-positive labelling of the pharyngeal mesenchyme and cartilages of the hyobranchial apparatus of the axolotl larvae, including ceratobranchials, ceratohyals, hypobranchials, and hypohyals, as well as the lower and the upper jaws (Fig. $1 \mathrm{C}-\mathrm{H}$ ). The difference between
Fig. 2. Results of one-sided head lateral plate mesoderm transplantations. (A) Schematics demonstrating grafting of GFP + head LPM (including all the pharyngeal mesoderm) from a GFP+ early somitogenesis stage (green, stage 20) into a white $(d / d)$ host. (F) Schematics demonstrating transplantation of a smaller area of the head LPM approximately $1 / 3$ of the area in the previous experiment. In both experiments the GFP+ LPM of the size shown in schematics was grafted into a white host whose corresponding mesodermal areas on one side had been removed before. (B) Schematics summarizing the results of two experiments, (green-elements marked with GFP after LPM transplantation (basibranchial 2) and heart myocardium, blue - all the rest elements of the hyobranchial apparatus, which has never been marked). (C, D,E) Transverse sections through the hyobranchial region of a representative 1 monthold juvenile after whole head LPM transplantation. Section planes are shown at $B$ with dashed lines. Note inclusion of the mesodermal GFP + cells into the basibranchial $2(D, E)$ on the side of transplantation, but not to basibranchial 1 (C). Green bright cells to the left of bbr1 and bbr2 represent connective tissue of the flank, which arises from LPM mesenchyme. (G) Frontal section through the basibranchial 2 in a 1 months old juvenile (section plane is shown at $B$ as a black dashed rectangular G); mesoderm contributes to this element through its length as seen by GFP+ labelling on the side of transplantation, (H) Frontal section through the caudal aspect of the basibranchial 2 and the heart anlage both labelled with GFP+ cells (section plane is shown at $B$ as a black dashed rectangular $H$ ), $(\mathbf{I}, \mathbf{J})$ Transverse section through the middle part of the hyobranchial apparatus as shown at (B) with a dashed line; ( $\mathbf{J})$ enlarged area shown with the dashed white rectangular framed at I, GFP+ mesodermal marking is noticeable only among the connective tissue of the flank and again, as in the previous experiment, in basibranchial 2 at the side of transplantation, but not in any other cartilaginous hyobranchial element. Abbr. as in Fig. 1. Scale bars: $100 \mu \mathrm{m}$.
A

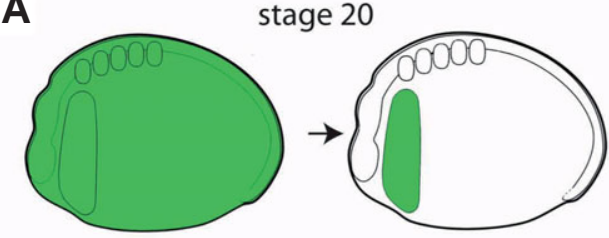

one-sided head mesoderm grafting
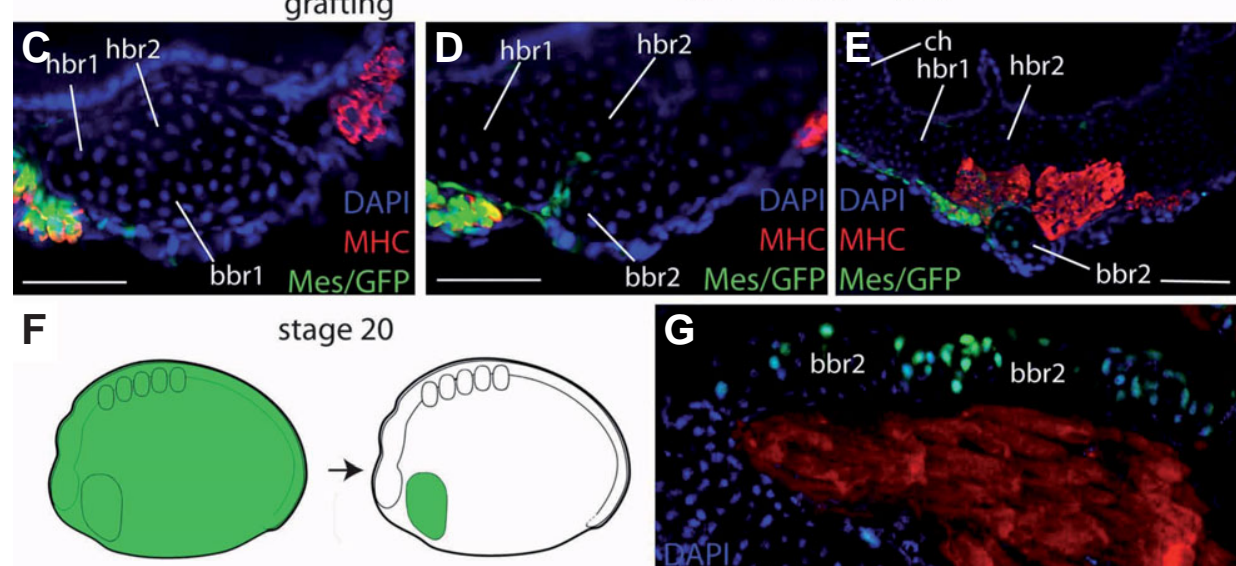

one-sided grafting of the lower part of the head mesoderm
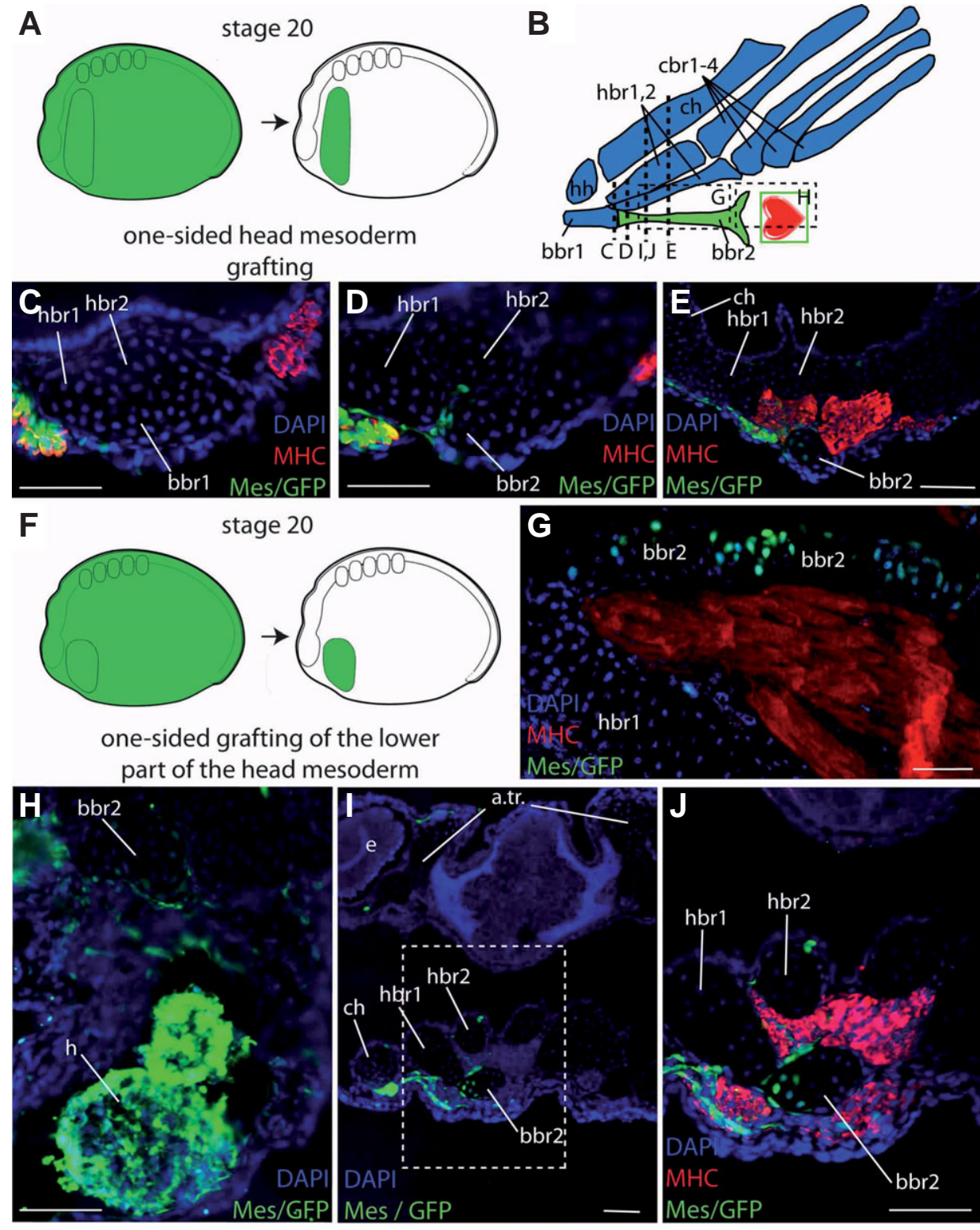
the bilateral and unilateral transplantations was in that in the latter experiments some GFP+ cells still partially labelled the cartilages on the side contralateral to the transplantation, while cartilaginous elements on the side of transplantation had some focal deficiencies in labelling (Fig. $1 \mathrm{I}, \mathrm{J})$. No such deficiencies were noted if transplantations were double sided. These results suggested that NC cells from both neural folds are required for the normal formation of the paired cartilages. The only cartilage of the viscerocranium, which was found completely not labelled in either experiment, was the basibranchial 2 (Fig. 1 C,D,H). NC cells were not found also in the posterior part of the trabeculae cranii (but labelled anterior trabeculae craniad to the optic nerve, Fig. 1I), the otic capsules, and the occipital bones (not shown).

\section{The head lateral plate mesoderm is the common source for the basibranchial 2 and the heart}

Due to medio-ventral position of the basibranchial 2 cartilage left unmarked in the previous experiment we considered head LPM as the most probable source for the skeletogenic mesenchyme, which forms this branchial cartilage. To check this possibility we first grafted homotopically and unilaterally large pieces of the head LPM from GFP+ donors to white (d/d) hosts at stage 20 embryos

A

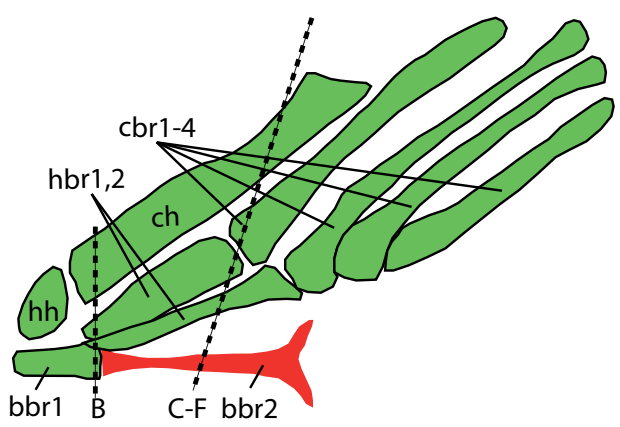

bbr1 B C-F bbr2
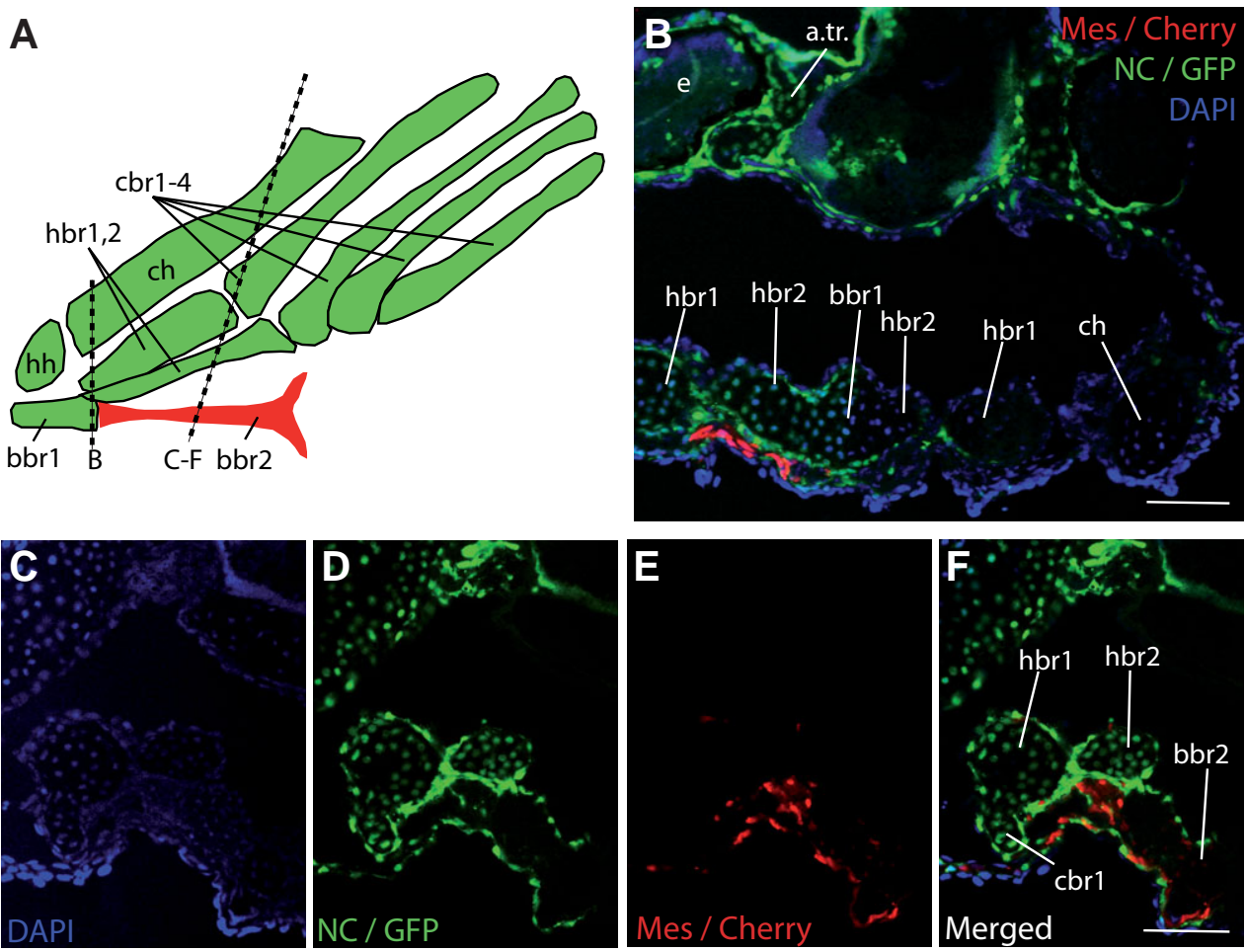

Fig. 3. Results of one sided double labelling of the neural crest (see Fig. 1B) and the head lateral plate mesoderm (see Fig. 2F). Two types of transplantations of tissues from a coloured donor to the same white host were done one by one with the interval of ca. 24-36 hours resulting in GFP+ labelling of nearly all the neural crest derivatives and Cherry+ labelling of head lateral plate mesoderm derivatives on the side of transplantation. (A) Schematics, summarising the results of the double labelling. Only basibranchial 2 is composed of mesoderm cells, while the rest of the hyobranchial apparatus is of neural crest origin. (B) Transverse section through the cranial part of the hyobranchial apparatus at the level of the basibranchial 1. Section plane see at A. (C-F) Nearly transverse section through a more caudal part of the hyobranchial apparatus at the level of the basibranchial 2. Note that neural crest GFP+cells and mesoderm Cherry+ cells never mix within I of the any of the cartilages forming distinct territories within the hyobranchial apparatus. Abbr. as on Figs. 1 and 2. Scale bars: $100 \mu \mathrm{m}$.
(Fig. 2A) avoiding transplantation of both endoderm and paraxial mesoderm. These grafts included nearly the whole head LPM (the mesodermal sheet, and from the most anterior extension of the mesodermal sheet just behind the forming eye cranially to the level cartilages, those revealed as NC-derived in the previous set of experiments, remained completely unlabelled on the side of the splantation, although we found GFP+ cells in the connective elements (Fig. 2 B-E). The only exception was the basibranchial 2 , which was labelled on the side of the transplantation (in 4 out of 4 examined transgenic chimeras) throughout its length (Fig. 2 fouble labelling of the NC (unilateral GFP+ neural fold f five embryos succio Of five embryos successful operations were made in two resulting basibranchial 2 on the side of operation and GFP+ labelling of the rest of the hyobranchial apparatus. Again no cells of mesodermal origin were found at the territory of the $\mathrm{NC}$, and vice versa, the NC cells did not mix to mesoderm to form basibranchial anlagen, always leaving clear borders between the elements of NC and of mesodermal origin (Fig. 3).

Taking into account ventral medial position of the basibranchial 2, it was quite reasonable to suggest that the most ventral part of the head LPM on each side of the embryo can be the source for the basibranchial2. To prove this assumption we further grafted the most ventral portion of the GFP+ head LPM approximately one third of the size of the transplant in the previous experiment (Fig. 2F). As expected, we found basibranchial 2 fully labelled on the side of transplantation in all cases (7 out of 7 evaluated chimeric larvae, Fig. 2 G-J). In all chimeras the heart myocardium was also labelled with GFP+ cells (Fig. $2 \mathrm{H})$. Thus, the most ventral part of the head LPM, which normally gives rise to the heart, is indeed the region of the head LPM, which have also a skeletogenic potential and contribute to the basibranchial 2 .

Since in our transplantation experiments we always found the labelling of a half of basibranchial 2 at the side of transplantation, we further transplanted GFP+ head LPM to one side of the white embryo, and Cherry+ head LPM 
to the other side of the same white embryo $(\mathrm{N}=3)$. Since this kind of operation caused a large wound on the belly of the operated embryos, only in one embryo the wound healed properly allowing medial structures to develop. This experiment resulted in one side of the basibranchial 2 labelled with GFP+ and the other side-with Cherry+ cells (not shown). A few cells from the contralateral sides intermingled and did not form sharp boundary, but there were no unlabelled cells in the cartilage.

\section{Discussion}

\section{Amphibian hyobranchial apparatus is of dual origin}

Results of our experiments, using transgenic axolotl system, a most modern and precise way of labelling living cells in amphibian embryos available to date, revealed dual origin of the hyobranchial apparatus of an urodelan amphibian, the Mexican axolotl ( $A$. mexicanum). With our transplantations of the prospective neural crest from all possible cranio-caudal regions and on one or both sides of the body from GFP+ tissue donors to white hosts, we did not find any single NC cell within the cartilage of the basibranchial 2 in any of our transgenic chimeras. In contrast, the grafting of the head LPM from transgenic donors to white hosts revealed the basibranchial 2, but not any other hyobranchial cartilage, was labelled.

Our fate mapping study confirmed a very old notion based on merely morphological discrimination of cells of NC and of mesodermal origin or NC extirpation experiments that some cartilages of the hyobranchial apparatus in amphibians may be not of NC, but of mesodermal origin. This was suspected by several authors not only for urodelans, e.g., Ambystoma, but also for anuran amphibians, such as Rana and Xenopus (Hörstadius, 1950, Raven, 1931, Sadaghiani and Thiebaud, 1987, Seufert and Hall, 1990, Stone, 1929). L.S. Stone was the first who suspected mesodermal origin of the basibranchial 2 in A. punctatum, as well as basihyal (= copula 1) cartilage and the hind portion of basibranchial (= copula 2) in $R$. pallustris (Stone, 1926, Stone, 1929). More recently, P. Chibon was not able to mark basibranchial 2 in Pleurodeles waltlii by means of ${ }^{3} \mathrm{H}$-thymidine autoradiography, when pieces of neural folds from different levels of a thymidine-treated open neural fold stage embryos were transplanted to normal host embryos (Chibon, 1967). Similarly, Sadaghiani and Theibaud (Sadaghiani and Thiebaud, 1987) approached the fate-mapping of the NC in Xenopus with interspecies tissue grafting from $X$. borealis to $X$. laevis, and consequent tissue discrimination by quinacrine nuclear staining specific for $X$. borealis. After hyoid or branchial NC segment transplantation they found a mixture of "donor" and "host" cells in the ceratohyal and branchial cartilages, and only "host" material in the basihyal (Sadaghiani and Thiebaud, 1987). However, the authors' overall conclusion of a mixture of mesodermal and NC cells in composing the hyobranchial cartilages could hardly be drawn from these data. This is because transplantation of a small portion of only one neural fold does not allow interpretation of the non-labelled host cells on sections as "non-crest" or specifically "mesodermal", because they may originate from host NC of other cranio-caudal levels or the contralateral host neural fold, which was not transplanted. Our unilateral transplantations of the GFP+ neural fold clearly show this situation resulting in that some hyobranchial elements represent mosaic marked and non-marked cartilage on the side of transplantation, as well as demonstrating inclusion of donor cells to the contralateral cartilages on the "control" side due to NC cell migration (see Fig. 1).

Aspecial case was the transplantation experiment in Ambystoma punctatum, when the head LPM was transplanted to the place of previously excised trunk somites (Stone, 1932). In 9 out of 17 cases, formation of an ectopic rod-like cartilage was observed. In contrast, an earlier analogous experiment in $R$. palustris did not reveal in a single case a slightest trace of cartilage in the transplanted tissues, suggesting that all the cartilages of the branchial arches in this species develop from NC, not from the underlying mesoderm (Stone, 1929). This was in apparent contradiction to the arguments of the same author based on morphological and NC extirpation experiments in Rana (Stone, 1929). Our NC and mesoderm transplantations in axolotl embryos gave ubiquitous and complementary results in all grafting series, demonstrating patterns similar to those predicted by earlier morphological/extirpation/ ectopic transplantation studies in several species of amphibians; hence, it is quite safely to say that most probably both urodelan and anuran modern amphibians have hyobranchial apparatus of dual origin. The extent of contribution of the mesoderm to this system may probably vary including one or more midline elements. In contrast to predictions based on NC marking in the previous reports (Sadaghiani and Thiebaud, 1987, Stone, 1926, Stone, 1929) we, however, did not reveal mesodermal cells in ceratohyals or ceratobranchials in axolotl, and we assume that this may hardly be so in other amphibians.

\section{Heart field pharyngeal mesoderm contributes to the basibran- chial 2 cartilage}

In our experiments, we initially transplanted large portions of the head LPM strictly avoiding transplantation of the paraxial head mesoderm (see Methods). Despite such an extensive grafting, only one cartilage appeared to be consistently marked with the GFP+ cells, suggesting that the head LPM has limited capacities to form skeletal tissues (in contrast to the head paraxial mesoderm, see: Sambasivan et al., 2011). Curiously, a somewhat similar result of limited cartilage-forming capacities of the head LPM was shown by transplantation of the head pharyngeal mesoderm to the region of trunk somites (Stone, 1932). In those experiments only one small (40 to $100 \mu \mathrm{m}$ in length) cartilage developed ectopically after transplantation of a relatively large area of LPM. In all cases of successful development of such an ectopic cartilage the ectopic heart developed nearby, although in four other cases the ectopic heart developed alone. Such a correlation between the development of the ectopic heart and cartilage thus indicated a relative proximity of the two anlagen in the graft (Stone, 1932). In our experimental series we performed homotopic transplantations of a smaller, the most ventrally positioned area of the head LPM (see Fig. 2A and Methods). In all resulting chimeras the heart was consistently labelled with GFP + cells along with the basibranchial 2 , suggesting that both the heart and the basibranchial 2 develop from a common mesodermal region.

Heart and major arteries nearby are the potential place for development of ectopic cartilages in amniotes (Lopez et al., 2003). These heart cartilages have, however, NC, but not the mesoderm origin at least in birds and mammals (Sumida et al., 1989). Although axolotl belongs to amphibians, the proximity of the anlagen of the heart and the prospective basibranchial 2, both of which show, or may show in certain conditions, chondrification of mesenchymal cells of different embryonic origin, may indicate a possibility of a 
common signaling event in the heart-field mesoderm, leading to cartilaginous differentiation of both NC derived ectomesenchyme and LPM. This is possible because the skeletogenic molecular programms are conserved among tissues of different origin in the head and in the trunk (Eames and Helms, 2004). Formation of cartilage in the heart and elsewhere from tissues of different embryonic origin is under control of common transcription factors, such as Sox9 (Lincoln et al., 2007, Lincoln et al., 2006). Cranial NC has wide skeletogenic potential, forming both cartilage, endochondral bone, or intramembranous ossification, but needs contact to pharyngeal endoderm and mesoderm to start skeletogenic differentiation (Seufert and Hall, 1990). Interestingly, the basibranchial 2 and the heart lie not only in close proximity, they both belong to the zone of expression of the Hox3 orthologs, which express in all three tissues of potential importance: heart mesoderm, cardiac NC and pharyngeal endoderm (Diman et al., 2011, Medeiros and Crump, 2012). To the best of our knowledge, formation of basibranchial 2 from the heart field LPM is the first case unequivocally demonstrating skeletogenic potential of the LPM in the head.

\section{Interchangeable contribution of the neural crest and meso- derm to the head skeleton: developmental and evolutionary implications}

Data from several model systems supported the view that pharyngeal cartilages originate exclusively from NC in vertebrates. Particularly, the genetic labelling of the NC in fish revealed that virtually all the elements of the pharyngeal arches, including all the basibranchial elements originate from this enigmatic germ layer (Kague et al., 2012, Mongera et al., 2013, Schilling and Kimmel, 1994). A series of reports from several laboratories in 70s-90s of the $\mathrm{XX}$ century on $\mathrm{NC}$ contribution to the skull in avian embryos based on chick-quail chimerisation studies congruently showed that the majority of the viscerocranium (with only possible exception to columella) of birds also originates from NC (reviewed by Gross and Hanken, 2008). Interestingly, mammalian laryngeal cartilages (thyroid, cricoid, and arythenoid cartilages), which are developing from $4^{\text {th }}$ to $6^{\text {th }}$ branchial arches, as well as the rest of the branchial and jaw skeleton, e.g., hypoglossal and mandibular cartilages, were also reported as NC derived (Chai et al., 2000, Matsuoka et al., 2005).

Our finding that the cartilaginous element (basibranchial 2) of the axolotl branchial arches develop from mesoderm turns us to discussion of interchangeable contribution of mesoderm and NC to the same (considered homologous) parts of the skeleton in development and evolution. As it was explicitly shown by Schneider (1999), NC can substitute the paraxial mesoderm in the avian head skeleton. Ectopic transplantation of a portion of the quail presumptive NC to the chick embryo in position, which is normally occupied by paraxial head mesoderm, resulted in development of chimeras with cranial bones of orbitotemporal region, composed of mesoderm and NC cells, morphologically indistinguishable from the normal chick bones, composed exclusively by mesodermal cells (Schneider, 1999). Furthermore, it seems that NC-derived mesenchyme could adequately respond to similar cues, which normally drive the skeletogenesis and proper patterning of the mesoderm-derived skeletal elements (Kuratani, 2005, Schneider, 1999). The mesodermal origin of a basibranchial element shown in our report demonstrates a somewhat reverse situation, when the mesodermal tissue contributes to the part of the head skeleton, which is otherwise expected to be NC-derived. The difference to the above mentioned situation is that we see this interchange in evolution, rather than in an experiment, with the mesoderm substitution of the NC in amphibians if compared to fish, birds, and mammals.

\section{Materials and Methods}

\section{Animals}

Adults of the Mexican axolotl (A. mexicanum) of $\mathrm{d} / \mathrm{d}$ white line, GFPand Cherry-nuclear transgenics (Kragl et al., 2009, Sobkow et al., 2006) were bred in the facility of the Max-Planck-Institute of Molecular Cell Biology and Genetics in Dresden, Center for Regenerative Therapies, TU Dresden, and aquarial of the Faculty of Biology, St. Petersburg State University, Russia. Eggs were kept in tap water at room temperature or, to delay development and synchronize clutches, at 4-8ㄷ․ Embryos and larvae were staged according to normal tables (Bordzilovskaya etal., 1989).

\section{Transgenesis and transgenics}

The generation of transgenic animals ubiquitously expressing GFP under the control of the CAGGS promotor has been described elsewhere (Sobkow et al., 2006). Using the same protocol, transgenic animals expressing nuclear Cherry under the control of the CAGGS promotor were generated as described (Kragl et al., 2009). The transgenic embryos, used as donors for operations, as well as the host embryos, had $\mathrm{d} / \mathrm{d}$ (white mutant) background. The $\mathrm{d} / \mathrm{d}$ mutant axolotls were chosen for better visualization of the fluorochromes in the cells. Justification for the use of this mutant as a basis for transgenic lines for the study of neural crest contribution to the skeletal tissues has been given in our recent publication (Epperlein et al., 2012).

\section{Embryo operations}

Before dejellying eggs were washed in $70^{\circ}$ ethanol to sterilize the jelly coat surfaces and then were dejellied in sterile $1 \times$ Steinberg solution containing antibiotics (Antibiotic-Antimycotic; Invitrogen, Karlsruhe, Germany) plus Fortum or Cyprofloxacim antibiotics. The embryos were then transferred into agar dishes (2\% agar in autoclaved tap water) filled with sterile Steinberg solution and arrested in deepenings of the agar layer. Operations were carried out with tungsten or preparation needles either in $4 \times$ Steinberg solution in order to obtain an optimal separation of tissue layers (epidermis, mesoderm, endoderm) in most cases or in 1× Steinberg solution, when an operation (e.g., grafting long bilateral neural folds) lasted 20-60 min. With hypertonic Steinberg solution tissue layers can be separated more easily.

\section{Neural fold (neural crest) grafting}

An unilateral (left) fragment of the most anterior head to the most posterior trunk neural fold $(n=5)$ containing $N C$, or the entire left and right cranial and trunk neural folds of a GFP+ donor $(n=5)$ were grafted into a white $(\mathrm{d} / \mathrm{d})$ host at stage 16 (Bordzilovskaya et al., 1989) where similar sized neural fold areas had been removed before. The implanted fold fragments were pressed against the body of the host with a piece of glass to assist healing. Grafting of the one neural fold fragment was carried out in $4 x$ Steinberg solution whereas the operation with two folds was done in $1 \times$ Steinberg, because it takes too long as that the embryos would survive if operated in $4 \times$ Steinberg. The estimated length of double neural folds grafted from GFP+ donors to white hosts gives ca. $95 \%$ of the neural folds of the embryo. This is approximately the same amount as the number of cells of the NC, which can be labelled in this way of grafting. See also our previous report for the extent of labelling of target tissues (Epperlein et al., 2012). Larvae were fixed at age of three weeks to two months. Larvae carrying two grafted folds were especially carefully examined on ca. 300 transverse and sagittal cryostat sections (see 
below). There was no evidence of GFP silencing in NC derivatives, such as dorsal root ganglia.

\section{Mesoderm grafting}

Operations on mesodermal tissues (head LPM, the ventral part of the head LPM) were generally performed as follows. The epidermis of one GFP+, or one Cherry+, or two fluorescent donors, and one white host embryos (all the same stage) was lifted in the left anterior trunk in a rectangular area reaching from the ventrolateral body to the neural tube. The mesodermal tissue was removed in the host as a single sheet and substituted with the mesoderm from the corresponding areas of the donor. Following implantation of the tissue from fluorescent donor(s), the host epidermis was folded back and pressed against the graft with a piece of glass for about 5 min to prevent curling. The $4 \times$ Steinberg solution was then diluted with distilled water to about the normal concentration. The embryos were allowed to develop into larvae (2-3 weeks).

1. Transplantation of the head LPM. Grafts from GFP+ donors to white (d/d) hosts at stage 20 embryos included all the lateral mesoderm from the line of somites and presomitic paraxial head mesoderm dorsally to the most ventral extension of the mesodermal sheet, and at least from the level of the developing eye cranially to the level of the fore-boundary of the somite 3 caudally. We have taken particular care not to transplant paraxial head mesoderm, which gives rise to some head muscles and hind portions of the neurocranium. This was checked by observing no GFP+ mesoderm in occipital bones and posterior trabeculae. Nine operations were made, of resulting chimeras five were analysed, of which in one case it was appeared that the donor and recipient were mixed up after the operation; the latter being discarded.

2. Transplantation of the most ventral part of the head LPM from one side GFP+ donors to white (d/d) hosts at stage 20 embryos. These grafts included the ventral one-third or less of the transplant taken in the previous experiment.

3. Transplantation of the head LPM from a GFP+ donor to one side of the white embryo, and Cherry+ donor to the other side of the same white embryo $(\mathrm{N}=3)$ was especially difficult due to bad healing of the wound. The ectoderm was first cut on the belly of the host and on both sides of the embryo was opened as ectodermal pockets; the head LPM of both sides of the body was excised from underneath the opened ectoderm and substituted with two (GFP+ and Cherry+) separate grafts. The yolk plug and the ectoderm hardly healed, therefore only in one embryo the ventral medial structures formed and were able to analyse.

4. Transplantation of one neural fold (GFP+) and the head LPM (Cherry+). The first step-an unilateral (left) neural fold of a GFP+ donor was grafted into a white $(d / d)$ host at stage 14-16 where the neural fold had been removed before as described above. When the embryos reached the stage 20, they were transplanted the ventral part of the head LPM from Cherry+ donors as described above.

\section{Sectioning and immunostaining}

Transverse cryosections $(20-25 \mu \mathrm{m})$ were cut through the shoulder region of the anterior trunk in about 1.5-2.5 month old juveniles that contained GFP+ tissues. Specimens were fixed with $4 \%$ paraformaldehyde at $4^{\circ} \mathrm{C}$ over night, washed in PBS, incubated in $20 \%$ sucrose overnight, infiltrated with $7.5 \%$ gelatine (AppliChem a1693,0500) overnight, embedded into $15 \%$ gelatine and frozen on dry ice. Cryosections were stained with primary antibodies against GFP (Invitrogen) and Cherry (clone 700-A103, Max Planck Institute of Molecular Cell Biology and Genetics, Dresden, antibody facility) to increase the visibility of transgenic donor cells. Alexa 488-conjugated secondary antibodies were used for enhancing GFP fluorescence and Cy3, Alexa 555 or Alexa 546-conjugated secondary antibodies were used for enhancing Cherry fluorescence. Additionally, we used rhodamine-conjugated anti-Myosin heavy chain antibodies (clone 4A.1025, a kind gift from Simon Hughes, Kings College, London) to visualize skeletal muscles. All sections were stained with DAPI, embedded into glycerol-PBS (1:1) and analysed with epifluorescence microscopes.
Some images were taken using multifoton confocal microscope (Leica TCS SP5 MP).

\section{Acknowledgements}

This work would never have started without the generous help and support of Prof. Elly M. Tanaka (Center for Regenerative Therapies, University of Technology, Dresden) and members of her team, especially Prof. Hans Epperlein, for parts of the grafting experiments and transferring technologies during the most critical time of establishing our new laboratory in Russia. We thank Arslan Valeev and Elena Kuternitskaya, as well as Haino Andreas and Marita Schütz, for their excellent technical assistance in breeding and taking care of animals in correspondingly, St. Petersburg and Dresden laboratories, and Anastasia Varlamova for evaluation of completeness of transgenesis of F2 Cherry+/- axolotls in the St. Petersburg's colony. We are grateful to Dr. Alexey Smirnov (St. Petersburg State University) for helpful advice on fluorescent imaging; Pavel Zykin, Lilia Hatmullina, and Nikolaj Kostin from Research Resource Centre "Molecular and cell technologies" (St. Petersburg State University; www.biomed.spbu.ru/en/) for assistance with confocal imaging and channel unmixing. This work was supported by the Alexander von Humboldt Foundation (Equipment donation, Zeiss SteREO Lumar.V12, grant No. 3.4-8151/07074 to YM) and DAAD Inter-Institutional Partnership Program (to Elly Tanaka and YM).

\section{References}

BORDZILOVSKAYA, N.P., DETLAFF, T.A., DUHON, S.T. and MALACINSKI, G.M. (1989). Developmental-stage series of axolotl embryos. In Developmental Biology of the Axolotl, (ed. ARMSTRONG, J. B. and MALACINSKI, G. M.). Oxford University Press, pp. 201-219.

CHAI, Y., JIANG, X., ITO, Y., BRINGAS, P., HAN, J., ROWITCH, D.H., SORIANO, P., MCMAHON, A.P. and SUCOV, H.M. (2000). Fate of the mammalian cranial neural crest during tooth and mandibular morphogenesis. Development 127: 1671-1679.

CHIBON, P. (1967). Marquage nucleaire par la thymidine tritiee des derives de la crete neurale chez FAmphibien Urodele Pleurodeles waltlii Michah. J Embryol Exp Morphol 18: 343-358.

DIMAN, N.Y.S.-G., REMACLE, S., BRTRAND, N., PICARD, J.J., ZAFFRAN, S. and REZSOHAZY, R. (2011). A retinoic acid responsive Hoxa3 transgene expression in embryonic pharyngeal endoderm, cardiac neural crest and a subdomain of the second heart field. PLOS ONE 6: e27624.

EAMES, B.F. and HELMS, J.A. (2004). Conserved molecular program regulating cranial and appendicular skeletogenesis. Dev Dyn 231: 4-13.

EPPERLEIN, H.-H., KHATTAK, S., KNAPP, D., TANAKA, E.M. and MALASHICHEV, Y. (2012). Neural crest does not contribute to the neck and shoulder in the Axolot (Ambystoma mexicanum). PLOS ONE 7: e52244.

GROSS, J.B. and HANKEN, J. (2008). Review of fate-mapping studies of osteogenic cranial neural crest in vertebrates. Dev Biol 317: 389-400.

HÖRSTADIUS, S. (1950). The Neural Crest. Its properties and derivatives in the light of experimental research. Oxford University Press, London, New York, Toronto.

KAGUE, E., GALLAGHER, M., BURKE, S., PARSONS, M., FRANZ-ODENDAAL, T. and FISCHER, S. (2012). Skeletogenic fate of zebrafish cranial and trunk neural crest. PLOS ONE 7: e47394.

KRAGL, M., KNAPP, D., NACU, E., KHATTAK, S., MADE, M., EPPERLEIN, H.-H. and TANAKA, E.M. (2009). Cells keep a memory of their tissue origin during axolot limb regeneration. Nature 460: 60-65.

KURATANI, S. (2005). Craniofacial development and the evolution of the vertebrates: the old problems on a new background. Zool. Sci. 22: 1-19.

LANDACRE, F.L. (1921). The fate of the neural crest in the head of the urodeles. $J$ Comp Neurol 33: 1-43.

LINCOLN, J., KIST, R., SCHERER, G. and YUTZEY, K.E. (2007). Sox9 is required for precursor cell expansion and extracellular matrix organization during mouse heart valve development. Dev Biol 305: 120-132.

LINCOLN, J., LANGE, A.W. and YUTZEY, K.E. (2006). Hearts and bones: shared regulatory mechanisms in heart valve, cartilage, tendon, and bone development. Dev Biol 294: 292-302 


\section{A. Davidian and Y. Malashichev}

LOPEZ, D., DURAN, A.C., DE ANDRES, A.V., GUERRERO, A., BLASCO, M. and SANS-COMA, V. (2003). Formation of cartilage in the heart of the spanish terrapin, Mauremys leprosa (Reptilia, Chelonia). J Morphol 258: 97-105.

MATSUOKA, T., AHLBERG, P.E., KESSARIS, N., IANNARELLI, P., DENNEHY, U., RICHARDSON, W.D., MCMAHON, A.P. and KOENTGES, G. (2005). Neural crest origins of the neck and shoulder. Nature 436: 347-355.

MEDEIROS, D.M. and CRUMP, J.G. (2012). New perspective on pharyngeal dorsoventral patterning in development and evolution of the vertebrate jaw. Dev Biol 371: 121-135.

MONGERA, A., SINGH, A.P., LEVESQUE, M.P., CHEN, Y.-Y., KONSTANTINIDIS, P. and NÜSSLEIN-VOLHARD, C. (2013). Genetic lineage labeling in zebrafish uncovers novel neural crest contributions to the head, including gill pillar cells. Development 140: 916-925.

RAVEN, C.P. (1931). Zur Entwicklung der Ganglienleiste. I. Die Kinematik der Ganglienleistenentwicklung bei den Urodelen. Arch EntwMech Org 125: 210-292.

REILLY, S.M. and LAUDER, G.V. (1988). Atavisms and the homology of hyobranchial elements in lower vertebrates. J Morphol 195: 237-245.

SADAGHIANI, B. and THIEBAUD, C.H. (1987). Neural crest development in the Xenopus laevis embryo, studied by interspecific transplantation and scanning electron microscopy. Dev Biol 124: 91-110.

SAMBASIVAN, R., KURATANI, S. and TAJBAKHSH, S. (2011). An eye on the head: the development and evolution of craniofacial muscles. Development 138:2401-2415.
SCHILLING, T.F. and KIMMEL, C.B. (1994). Segment and cell type lineage restrictions during pharyngeal arch development in the zebrafish embryo. Development 120: 483-494.

SCHNEIDER, R.A. (1999). Neural crest can form cartilages normally derived from mesoderm during development of the avian head skeleton. Dev Biol208:441-455.

SEUFERT, D.W. and HALL, B.K. (1990). Tissue interactions involving cranial neural crest in cartilage formation in Xenopus laevis (Daudin). Cell Differ Dev32: 153-166.

SOBKOW, L., EPPERLEIN, H.H., HERKLOTZ, S., STRAUBE, W.L. and TANAKA E.M. (2006). A germline GFP transgenic axolotl and its use to track cell fate: dua origin of the fin mesenchyme during development and the fate of blood cells during regeneration. Dev Biol 290: 386-397.

STONE, L.S. (1926). Further experiments on the extirpation and transplantation of mesectoderm in Ambystoma punctatum. J Exp Zool 44: 95-131.

STONE, L.S. (1929). Experiments showing the role of migrating neural crest (mesectoderm) in the formation of head skeleton and loose connective tissue in Rana palustris. Roux'Arch. EntwMech Org 118: 40-77.

STONE, L.S. (1932). Transplantation of hyobranchial mesentoderm, including the right lateral anlage of the second basibranchium, in Amblystoma punctatum. $J$ Exp Zool 62: 109-123.

SUMIDA, H., AKIMOTO, N. and NAKAMURA, H. (1989). Distribution of the neural crest cells in the heart of birds: a three dimensional analysis. Anat Embryol 180: 29-35. 


\section{Further Related Reading, published previously in the Int. J. Dev. Biol.}

Expression of the cardiac actin gene in axolotl embryos

T Masi, M Drum, L Hall, R Dahnarajan and A D Johnson

Int. J. Dev. Biol. (2000) 44: 479-484

http://www.intjdevbiol.com/web/paper/11032182

The cardiac neural crest in Ambystoma mexicanum

N S Bashir and J B Armstrong

Int. J. Dev. Biol. (1999) 43: 269-274

http://www.intjdevbiol.com/web/paper/10410907

The in vivo and in vitro effects of bone morphogenetic protein-2 on the development of the chick mandible

$\mathrm{S}$ Ekanayake and B $\mathrm{K}$ Hall

Int. J. Dev. Biol. (1997) 41:67-81

http://www.intjdevbiol.com/web/paper/9074939

Urodele (e.g., axolotl) embryos in the undergraduate laboratory class: an essay describing a multifaceted learning experience

G M Malacinski and $\mathrm{H} M$ Chung

Int. J. Dev. Biol. (1996) 40: 901-905

http://www.intjdevbiol.com/web/paper/8877465

What insights into the phenomena of cell fate determination and cell migration has the study of the urodele neural crest provided?

$\mathrm{H}$ H Epperlein and $\mathrm{J}$ Löfberg

Int. J. Dev. Biol. (1996) 40: 695-707

http://www.intjdevbiol.com/web/paper/8877442

5 yr ISI Impact Factor $(2011)=2.959$
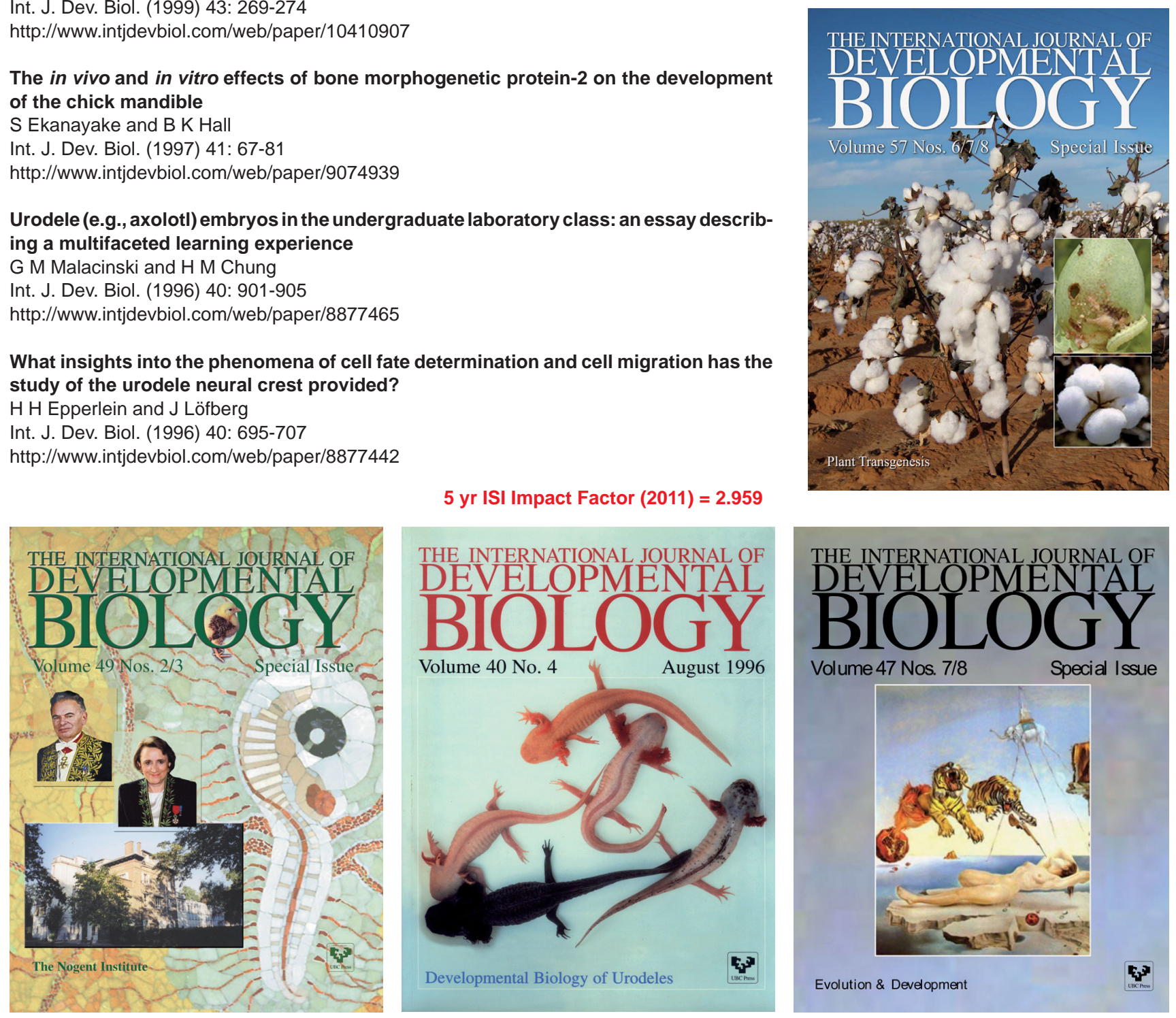

Volume 47 Nos. $7 / 8$
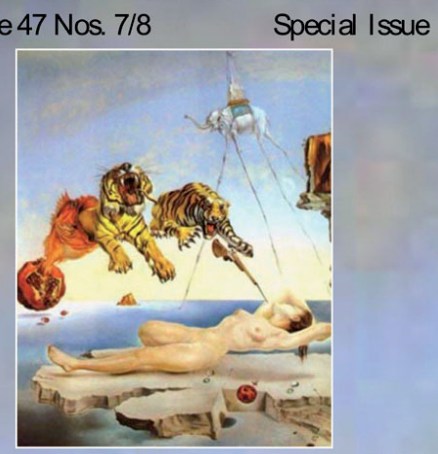

Developmental Biology of Urodeles 